\title{
The clinical, laboratory, and radiologic improvement due to siltuximab treatment in idiopathic multicentric Castleman's disease
}

\author{
Gi-June Min ${ }^{1}$, Young-Woo Jeon ${ }^{1}$, Sung-Soo Park ${ }^{1}$, Silvia Park ${ }^{1}$, Seung-Hawn Shin ${ }^{2}$, Seung-Ah Yahng ${ }^{3}$, \\ Jae-Ho Yoon ${ }^{1}$, Sung-Eun Lee ${ }^{1}$, Byung-Sik Cho ${ }^{1}$, Ki-Seong Eom ${ }^{1}$, Yoo-Jin Kim ${ }^{1}$, Seok Lee ${ }^{1}$, Hee-Je Kim ${ }^{1}$, \\ Chang-Ki Min ${ }^{1}$, Dong-Wook Kim', Jong-Wook Lee ${ }^{1}$, and Seok-Goo Cho ${ }^{1}$
}

\begin{abstract}
${ }^{1}$ Department of Hematology, Seoul St. Mary's Hematology Hospital, College of Medicine, The Catholic University of Korea, Seoul; ${ }^{2}$ Department of Hematology, Yeouido St. Mary's Hematology Hospital, College of Medicine, The Catholic University of Korea, Seoul; ${ }^{3}$ Department of Hematology, Incheon St. Mary's Hematology Hospital, College of Medicine, The Catholic University of Korea, Incheon, Korea
\end{abstract}

Received: October 4, 2019 Revised : October 23, 2019 Accepted: October 24, 2019

Correspondence to

Seok-Goo Cho, M.D.

Department of Hematology, Seoul St. Mary's Hematology Hospital, College of Medicine, The Catholic University of Korea, 222 Banpo-daero, Seocho-gu, Seoul o6591, Korea

Tel: $+82-2-2258-6052$

Fax: +82-2-599-3589

E-mail: chosg@catholic.ac.kr https://orcid.org/0000-00025429-4839
Background/Aims: Idiopathic multicentric Castleman disease (iMCD) comprises approximately $30 \%$ of all cases of Castleman disease. It is characterized by constitutional symptoms, enlarged lymph nodes at multiple anatomical sites, and laboratory test abnormalities, which are primarily related to the overproduction of interleukin 6 (IL-6). Siltuximab is a human-mouse chimeric immunoglobulin G1 $\kappa$ monoclonal antibody against human IL- 6 . In view of the limited treatment options for iMCD, this study aimed to evaluate the efficacy and safety of siltuximab in the management of this condition.

Methods: In this real-world retrospective study, we administered siltuximab to 15 patients with iMCD who previously received conventional chemotherapy and/or steroid pulse therapy. The median time to a durable symptomatic response was 22 days (range, 17 to 56). The serum hemoglobin and albumin levels and erythrocyte sedimentation rates significantly normalized after the first 3 months of siltuximab treatment. Lymph node involution, assessed using imaging, was relatively gradual, demonstrating a complete or partial response at 6 months.

Results: On an average, the improvements in clinical, laboratory, and radiologic parameters of iMCD in responders were observed after one, three, and eight cycles of siltuximab treatment, respectively. Siltuximab demonstrated a favorable safety profile, and prolonged treatment was well-tolerated.

Conclusions: Despite the small sample size of the present study, the results are encouraging and demonstrate the potential of siltuximab as the first-line treatment of iMCD. Further large multicenter studies are needed to evaluate the clinical outcomes and adverse events associated with siltuximab.

Keywords: Multi-centric Castleman's disease; Siltuximab; Interleukin-6; Tolerance

\section{INTRODUCTION}

Castleman disease (CD) was first described in the 1950 s as a rare nonmalignant lymphoproliferative disorder in patients with localized mediastinal lymphadenopathy
$[1,2]$. CD is classified as multicentric (MCD) or unicentric (UCD) based on its anatomical distribution; MCD constitutes approximately $30 \%$ of all CD cases with high mortality [3-6]. In the general population, the incidence of UCD and MCD is 15 and 5 per million patients per 
year, respectively, with regional variations [7].

MCD is characterized by systemic symptoms such as night sweats, fever, chills, fatigue, anorexia, anasarca, and cachexia. Its commonest features are enlarged lymph nodes at multiple anatomical sites, cytopenia, hypoalbuminemia, and increased concentrations of acute-phase proteins, such as fibrinogen and C-reactive protein (CRP) $[4,8]$. These apparently vague signs and symptoms result from the overproduction of interleukin 6 (IL-6), which plays a central role in the pathophysiology of MCD by inducing multiple processes such as the proliferation of B lymphocytes and plasma cells and the secretion of vascular endothelial growth factor (VEGF) [9].

Recent findings have elucidated the role of viral pathogenesis, particularly in human immunodeficiency virus (HIV)-related cases. Viral IL-6 may trigger the development of MCD in HIV-seropositive patients. However, most patients with MCD are HIV-negative, and the role of human IL-6 in HIV-negative MCD is well established $[8,10]$. MCD may be subdivided according to its etiology into human herpes virus (HHV)-8-associated MCD, polyneuropathy, endocrinopathy, monoclonal gammopathy, skin change-associated MCD, idiopathic MCD (iMCD), and MCD-like disorders [11]. The etiology of iMCD is unknown, and owing to its rarity, the options for its treatment are limited. In symptomatic iMCD, the treatment options include administration of high-dose steroids, rituximab, or combined conventional chemotherapy regimens such as cyclophosphamide, adriamycin, vincristine, and prednisolone (CHOP).

Siltuximab is a human-mouse chimeric immunoglobulin G1к monoclonal antibody against human IL-6. In a multicenter randomized and controlled phase 2 trial that included 79 HIV-negative iMCD patients, siltuximab with supportive care provided superior outcomes than supportive care alone, with tolerable safety and efficacy $[12,13]$. Furthermore, recently published consensus guidelines for the treatment of iMCD recommend siltuximab with or without corticosteroids as first-line therapy [14].

In this study, we determined the efficacy and safety of siltuximab in 15 patients with MCD, of whom two experienced sustained disease regression on long-term follow-up. The 15 patients were carefully examined to exclude the presence of HIV or HHV-8 infection, in ad- dition to other infectious diseases that mimic the signs and symptoms of MCD.

\section{METHODS}

\section{Patients and study design}

In this retrospective cohort analysis, we enrolled 15 patients with symptomatic iMCD who were eligible for siltuximab treatment between December 9, 2010 and October 31, 2018. Eligibility was based on detailed medical history, pathological diagnosis, and results of in-depth physical examinations, laboratory tests, and radiologic evaluation. The laboratory parameters examined from the time of diagnosis included the erythrocyte sedimentation rates (ESRs) and hemoglobin, CRP, creatinine, total protein, albumin, and $\beta 2$-microglobulin levels. The presence of Epstein-Barr virus infection was assessed using the real-time quantitative polymerase chain reaction (RQ-PCR). Radiological imaging included computed tomography (CT) of the neck, chest, and abdomen and positron emission tomography-CT; imaging was conducted at the time of diagnosis and every 3 months thereafter. In all cases of iMCD, the diagnosis was confirmed by a senior laboratory medicine physician with more than 10 years of experience. Pathological confirmation was based on lymph node biopsies and on the criteria established by a central pathology laboratory.

The symptoms at presentation were carefully assessed and classified individually based on the National Cancer Institute Common Terminology Criteria for Adverse Events (NCI-CTC-AE), version 4.0 and the Eastern Cooperative Oncology Group (ECOG) performance status score. Some of the patients had received prior treatment; however, none had prior exposure to IL-6-targeted therapy. Patients were excluded if they were HIV-seropositive, had any evidence of HHV-8 infection on RQ-PCR in plasma or paraffin-embedded tissue, had clear evidence of a significant infection (e.g., hepatitis B or C), or had concurrent lymphoma.

\section{Ethical approval}

All procedures performed in this study were in accordance with the ethical standards of the Institutional Review Board of Seoul at St. Mary's Hematology Hospital, Catholic University of Korea (KC19RESIo696) and 
the 1964 Helsinki declaration and its later amendments or comparable ethical standards. Informed consent was obtained from all individual participants included in the study.

\section{Treatment procedures and outcomes}

All patients received an intravenous infusion of siltuximab (11 mg/kg) at 3-week intervals along with best supportive care, which included management of effusions, infections, transfusions, and infusion-related reactions; it also involved the administration of antipruritics, antihistamines, antipyretics, and analgesics according to standard institutional guidelines. All patients continued treatment until treatment failure, which was defined as the appearance of new disease-related grade $\geq 3$ symptoms, persistence of grade $\geq 2$ disease-related symptoms for more than 3 weeks, elevated (by more than 1 point) ECOG scores persisting for at least 3 weeks, and radiological progression based on the modified Cheson criteria [15].

The primary endpoints included a durable symptomatic response and radiologic improvements for at least 3 months during treatment. Since there are no established criteria for evaluating iMCD, we used the modified Cheson criteria owing to the lymphoproliferative nature of iMCD.

\section{Statistical analysis}

The categorical variables were compared using the chisquare or Fisher's exact tests, and continuous variables were assessed using the Student's $t$ test or Mann-Whitney $U$ tests for comparison between the groups. Pearson analysis was employed for calculating the correlation coefficients. Statistical significance was set at $p<0.05$, and all $p$ values reported are two-sided. All statistical analyses were performed using the SPSS version 24.0 (IBM Inc., Chicago, IL, USA) software package.

\section{RESULTS}

\section{Baseline characteristics of the patients}

The median age of the cohort was 44 years (range, 24 to 76$)$; nine $(60.0 \%)$ patients were male. Overall, nine (60.0\%) patients had previously received corticosteroid pulse therapy or chemotherapy combined with corticosteroids prior to initiating siltuximab. A total of 10
(66.7\%) patients had an ECOG performance score of 2, mostly owing to the iMCD-related symptoms of fatigue $(\mathrm{n}=10,66.7 \%)$, malaise $(\mathrm{n}=9,60.0 \%)$, peripheral sensory neuropathy ( $\mathrm{n}=8,53.3 \%)$, sustained fever $(\mathrm{n}=7,46.7 \%)$, peripheral edema $(n=5,33.3 \%)$, and multiple palpable lymphadenopathies ( $n=4,26.7 \%)$.

Prior to siltuximab treatment, the CRP levels in eight (53.3\%) patients were above the normal limit (normal range, o to $5 \mathrm{mg} / \mathrm{dL}$ ), and all patients had an ESR exceeding $20 \mathrm{~mm} / \mathrm{hr}$ (normal range, o to 20 ); 10 (66.7\%) and four $(26.7 \%)$ patients presented with anemia (hemoglobin $<12.0 \mathrm{~g} / \mathrm{dL}$ ) and comorbidities related to renal impairment (creatinine $>1.2 \mathrm{mg} / \mathrm{dL}$ ), respectively. The median albumin to globulin ratio was 2.51 (range, 1.51 to 4.33). Based on the recent consensus diagnostic criteria for iMCD by Fajgenbau [16], all patients had enlarged lymph nodes (>1 cm in width) in at least two lymph node stations. On histopathologic review, all lymph node abnormalities were consistent with those of the iMCD spectrum; 14 (93.3\%) and one (6.7\%) patients had plasma cell and hyaline vascular subtypes, respectively. The baseline characteristics of the patients, including laboratory values, are summarized in Table 1.

\section{Clinical responses of the siltuximab-treated patients}

The median duration of siltuximab treatment was 9 months (range, 1 to 95 ); 11 patients (64.7\%) demonstrated significant symptomatic responses. The median number of days from the initiation of siltuximab to a durable symptomatic response was 22 days (range, 17 to 56 ). On laboratory evaluation after the first 3 months of treatment, the significant changes were noted in levels of hemoglobin (from 10.6 to $13.1 \mathrm{~g} / \mathrm{dL}$; $\mathrm{p}<0.01$ ), ESR (from 74.1 to $21.4 \mathrm{~mm} / \mathrm{hr} ; p<0.01$ ), serum total protein (from 8.5 to $7.4 \mathrm{~g} / \mathrm{dL} ; p=0.013$ ), and serum albumin (from 3.2 to $4.0 \mathrm{~g}$ / $\mathrm{dL} ; \mathrm{p}<0.01$ ) (Table 2).

Dramatic regression of multifocal lymphadenopathy was observed on CT during the first siltuximab infusion in one patient (Fig. 1); detailed analysis of the CT images of the neck, chest, and abdomen by a radiologist revealed complete remission (CR) and partial remission (PR) in three $(20.0 \%)$ and seven (46.7\%) patients, respectively (Table 3). The remaining three patients had stable disease (SD) with symptomatic relief; they continued treatment with siltuximab.

Two patients underwent long-term siltuximab infusion 
Table 1. Baseline characteris tics of the siltuximab-treated iMCD patients at the $\mathrm{CHH}$

\begin{tabular}{|c|c|}
\hline Characteristic & All patients $(n=15)$ \\
\hline Age, yr & $44(24-76)$ \\
\hline Men & $9(60.0)$ \\
\hline \multicolumn{2}{|l|}{ ECOG-PS score } \\
\hline 1 & $2(13 \cdot 3)$ \\
\hline 2 & $10(66.7)$ \\
\hline 3 & $3(20.0)$ \\
\hline \multicolumn{2}{|l|}{ Disease histology type } \\
\hline Hyaline vascular & $1(6.7)$ \\
\hline Plasma cell type & $14(93 \cdot 3)$ \\
\hline \multicolumn{2}{|l|}{ Disease-related symptom } \\
\hline Fatigue & $10(66.7)$ \\
\hline Malaise & $9(60.0)$ \\
\hline Peripheral sensory neuropathy & $8(53 \cdot 3)$ \\
\hline Sustained fever & $7(46.7)$ \\
\hline Peripheral edema & $5(33 \cdot 3)$ \\
\hline $\begin{array}{l}\text { Multiple palpable } \\
\text { lymphadenopathies }\end{array}$ & $4(26.7)$ \\
\hline \multicolumn{2}{|l|}{ Previous systemic treatment } \\
\hline $\begin{array}{l}\text { Corticosteroids with/ } \\
\text { without chemotherapy }\end{array}$ & $9(60.0)$ \\
\hline None & $6(40.0)$ \\
\hline Chemotherapy regimen & $7(46.7)$ \\
\hline R-CHOP, 6 cycles & $1(14 \cdot 3)$ \\
\hline CVP, 6 cycles & $3(42.8)$ \\
\hline
\end{tabular}

(95 and 90 months, respectively). Both had similar durable tumor and symptomatic responses that lasted for more than 7 years without disease relapse; they also demonstrated a sustained reduction in the number of iMCD-related symptoms and inflammatory markers, with maintenance of normalized hemoglobin levels. Fig. 2 shows the serum hemoglobin, CRP, ESR, total protein, and albumin values over 90 months in one of the two patients. Except for CRP, all the other parameters recovered during cycles 3 to 15 of siltuximab infusion. CRP normalization was delayed owing to recurrent infections in cycles 27 to 33 , which required long-term oral antibiotic therapy and regular evaluation by the infectious diseases department.

\section{Adverse events in siltuximab-treated patients}

Unfortunately, two (13.3\%) patients discontinued treat-

\begin{tabular}{|c|c|}
\hline Characteristic & All patients $(n=15)$ \\
\hline CHOP, 6 cycles & $2(28.6)$ \\
\hline $\begin{array}{l}\text { Etoposide, } 1 \text { cycle and CHOP, } \\
3 \text { cycles }\end{array}$ & $1(14 \cdot 3)$ \\
\hline Chemotherapy plus corticosteroids & $3(20.0)$ \\
\hline \multicolumn{2}{|l|}{ Laboratory characteristic } \\
\hline Hemoglobin, g/dL & $11.0(6.1-13 \cdot 3)$ \\
\hline C-reactive protein, $\mathrm{mg} / \mathrm{L}$ & $4.91(0.17-22.99)$ \\
\hline $\begin{array}{l}\text { Erythrocyte sedimentation rate, } \\
\mathrm{mm} / \mathrm{hr}\end{array}$ & $84(29-120)$ \\
\hline Creatinine, mg/dL & $0.89(0.33-1.66)$ \\
\hline Lactate dehydrogenase, g/dL & $275.5(166.0-1,290$. \\
\hline Total protein, serum, g/dL & $8.8(5 \cdot 3-11.3)$ \\
\hline Albumin, serum, g/dL & $3.4(2.1-3.9)$ \\
\hline$\beta_{2}$-Microglobulin, $\mu \mathrm{g} / \mathrm{mL}$ & $2.857(1.496-7.130)$ \\
\hline IgG, serum, mg/dL & $3,829(983-6,932)$ \\
\hline $\operatorname{IgA}$, serum, mg/dL & $483(82-2,295)$ \\
\hline IgM, serum, mg/dL & $160(91-1,065)$ \\
\hline IgE, serum, mg/dL & $1,256.5(15.2->10,000)$ \\
\hline \multicolumn{2}{|c|}{$\begin{array}{l}\text { Values are presented as median (range) or number (\%). } \\
\text { iMCD, idiopathic multicentric Castleman disease; CHH } \\
\text { Catholic Hematology Hospital; ECOG-PS, Eastern Coop- } \\
\text { erative Oncology Group-Performance Status; R-CHOP } \\
\text { rituximab, cyclophosphamide, doxorubicin, vincristine } \\
\text { prednisolone; CVP, cyclophosphamide, vincristine, prednis- } \\
\text { olone; CHOP, cyclophosphamide, doxorubicin, vincristine } \\
\text { prednisolone; Ig, immunoglobulin. }\end{array}$} \\
\hline
\end{tabular}

ment with siltuximab. Despite symptom improvement after the first infusion, one patient voluntarily discontinued treatment after the second infusion owing to old age, decreased performance, and poor general condition and was transferred to another hospital for best supportive care only. Interestingly, this female patient was diagnosed with biopsy-proven hyaline vascular-type iMCD. Hyaline vascular-type usually manifests as UCD and rarely requires systemic treatment [9], but this patient suffered from multiple lymphadenopathies, recurrent fever, weight loss, CRP elevation, hypoalbuminemia, and profound leukocytosis without evidence of systemic infection. We recognized this case as a rare multicentric manifestation of hyaline vascular-type Castleman's disease. The other patient presented with obstructive uropathy owing to a lymphoproliferative mass, which was 
compressing the right ureter; this patient achieved SD after four cycles of siltuximab. However, the mass did not regress, and the treatment regimen was altered from siltuximab to conventional chemotherapy (CHOP) despite significant symptomatic improvement and maintained renal function.

The commonest adverse event in the cohort was infection $(26.7 \%)$, mostly comprising mild and moderate upper respiratory viral infections, followed by maculopapular rashes (20.0\%), neuropathy of NCI-CTC-AE grade $\geq 2$ (13.4\%), and hepatopathy (13.4\%). Our study showed

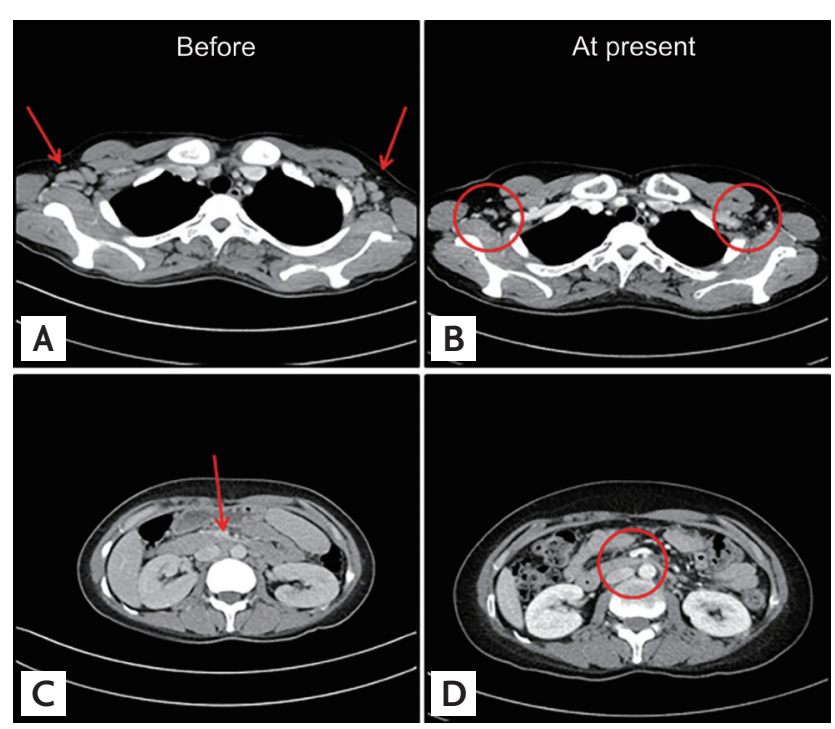

Figure 1. Significant regression of enlarged tissue masses after siltuximab infusion. Computed tomography (CT) findings in a representative patient with idiopathic multicentric Castleman disease. (A) CT before infusion. (B) Chest CT after infusion. (C) Abdominal CT before infusion. (D) Abdominal CT after infusion. Arrows demonstrating the location of the enlarged tissue mass in a representative patient. The CT scan results of a representative patient with idiopathic multicentric Castleman disease ere indicated by circles. similar profiles for the adverse events compared with the incidence rates of upper respiratory tract infection (36\%) and maculopapular rash (34\%) reported by van Rhee et al. [12]. We also observed that siltuximab is safe and well-tolerated during prolonged exposure in two patients for a period of 95 and 90 months, respectively. There was a profound symptomatic and laboratory disease remission during siltuximab maintenance, with only one experienced upper respiratory tract infection which required oral antibiotic therapy. No severe adverse events were observed. The clinical responses and adverse events in the cohort are summarized in Table 3.

\section{DISCUSSION}

This study reports real-world data based on the clinical experience with siltuximab in the treatment of symptomatic iMCD. We retrospectively assessed the efficacy and safety of siltuximab in HIV- and HHV8-negative Korean patients with iMCD in an actual clinical setting.

$\mathrm{CD}$ is a rare disease that comprises a heterogeneous group of lymphoproliferative disorders with characteristic histopathological appearances of lymph nodes ranging from atrophic germinal centers with hypervascularization to hyperplastic germinal centers with polytypic plasmacytosis [16]. Unlike UCD, which involves a single enlarged lymph node, has relatively mild symptoms, and is curable by lymph node excision, MCD involves multicentric lymphadenopathy, systemic inflammation and related constitutional symptoms, cytopenia, and potentially fatal multiorgan failure, all of which result from a cytokine storm including IL-6 [16]. The etiology of HHV8- and HIV-negative iMCD is unknown, and the charac-

Table 2. Laboratory responses after the first 3 months of siltuximab treatment

\begin{tabular}{lccc}
\hline Variable & Pre-treatment & Post-treatment & $p$ value \\
\hline Hemoglobin, g/dL & $10.6 \pm 2.3$ & $13.1 \pm 1.9$ & $<0.01$ \\
C-reactive protein, mg/dL & $6.49 \pm 1.17$ & $3.49 \pm 0.98$ & 0.219 \\
Erythroid sedimentation rate, $\mathrm{mm} / \mathrm{hr}$ & $74.1 \pm 30.0$ & $21.4 \pm 17.9$ & $<0.01$ \\
Creatinine, mg/dL & $0.93 \pm 0.37$ & $1.01 \pm 0.36$ & 0.226 \\
Lactate dehydrogenase, IU/L & $383 \pm 284$ & $408 \pm 140$ & 0.740 \\
Total protein, g/dL & $8.5 \pm 2.1$ & $7.4 \pm 1.0$ & 0.013 \\
Albumin, g/dL & $3.2 \pm 0.56$ & $4.0 \pm 0.59$ & $<0.01$ \\
\hline
\end{tabular}

Values are presented as mean $\pm \mathrm{SD}$. 
Table 3. Clinical responses of siltuximab-treated patients with MCD at the $\mathrm{CHH}$

\begin{tabular}{lc}
\hline Clinical response & All patients $(\mathrm{n}=15)$ \\
\hline Duration of treatment, mon & $9(1-95)$ \\
\hline Symptomatic response & $11(64.7)$ \\
Time to durable symptomatic response, & $22(17-56)$ \\
day & \\
\hline Imaging response ${ }^{\mathrm{a}}$ & \\
\hline Complete remission & $3(20.0)$ \\
\hline Partial remission & $7(46.7)$ \\
\hline Stable disease & $3(20.0)$ \\
\hline Siltuximab discontinuation & $1(6.7)$ \\
\hline Follow-up loss before imaging & $1(6.7)$ \\
response & \\
Clinical characteristic at 3 months & $13.8(10.1-5.4)$ \\
\hline Hemoglobin, g/dL & $0.99(0.01-21.0)$ \\
\hline C-reactive protein, mg/L & $18(2-61)$ \\
\hline Erythrocyte sedimentation rate, & $2(13.4)$ \\
\hline mm/hr & \\
\hline Creatinine, mg/dL & $0.89(0.43-1.62)$ \\
\hline Lactate dehydrogenase, g/dL & $391.0(236.0-751.0)$ \\
\hline Total protein, serum, g/dL & $4.1(3.0-5.0)$ \\
\hline Albumin, serum, g/dL & \\
\hline Adverse events & \\
\hline Upper respiratory infection & \\
\hline Maculopapular rash & \\
\hline
\end{tabular}

Values are presented as median (range) or number (\%). Toxicity was evaluated according to the NCI CTC-AE guidelines. MCD, multicentric; CHH, Catholic Hematology Hospital; NCI CTC-AE, National Cancer Institute Common Terminology Criteria for Adverse Events.

${ }^{a}$ Imaging responses were evaluated based on the Cheson criteria (selected owing to the lymphoproliferative nature of multicentric Castleman disease) for computed tomography images of the neck, abdomen, and chest, that were obtained at 6-month intervals.

${ }^{b}$ This patient had obstructive uropathy due to a lymphoproliferative mass compressing the right ureter. After four cycles of siltuximab, imaging showed stable disease without mass regression.

${ }^{\circ}$ This patient showed symptomatic improvement after the first siltuximab infusion. However, owing to old age, decreased performance, and a poor general condition, the patient voluntarily discontinued siltuximab after the second infusion, and was transferred to another hospital for best supportive care only. teristics of this disease are considerably less well understood than those of HHV-8-associated MCD.

A working group of 42 international experts recently established evidence-based consensus treatment guidelines for iMCD; these guidelines recommended siltuximab infusion (11 mg/kg) at 3-week intervals with or without corticosteroids as first-line therapy in all cases of iMCD [14]. This recommendation was based on the efficacy, safety, and patient responses to siltuximab, in addition to its global approval. The regimen involves continuation of siltuximab monotherapy for an indefinite period with tapering of corticosteroids. The disease severity in patients who fail to respond to siltuximab should be categorized based on disease status; severe iMCD may require intensive care and confers a high risk of multiorgan failure. According to the guidelines, patients with non-severe iMCD should receive 4 to 8 weekly doses of rituximab at a dose of $375 \mathrm{mg} / \mathrm{m}^{2}$ with or without corticosteroids or immunosuppressive agents such as cyclosporin, sirolimus, tacrolimus, thalidomide, or bortezomib. In patients with severe iMCD, weekly administration of siltuximab is recommended at a dose of $11 \mathrm{mg} / \mathrm{kg}$, with high-dose corticosteroids [14].

Overall, $64.7 \%$ of patients had a durable symptomatic response to siltuximab, with a median duration of response of 22 days (range, 17 to 56). The major laboratory indicators, including ESR, hemoglobin, and serum albumin concentrations, normalized rapidly within 3 months after treatment initiation. In contrast, lymph node involution, assessed using CT, occurred more gradually, with an overall response rate $(\mathrm{CR}+\mathrm{PR})$ of $66.7 \%$. In responders, the timelines for the clinical, laboratory, and radiologic iMCD improvements were significantly different, requiring an average of one, three, and eight cycles of siltuximab treatment, respectively. Therefore, we recommend performing laboratory tests every 3 months. Radiologic evaluation should be performed every 6 months because as radiologic confirmation of $\mathrm{CR}$ requires at least 6 months from the initiation of siltuximab treatment. Additionally, although many patients showed rapid symptomatic responses after siltuximab treatment, additional intensive chemotherapy regimens may be considered in cases where symptoms are correlated with the iMCD mass effect, which improves considerably slowly compared to common constitutional symptoms.

Our study has certain limitations owing to the retro- 

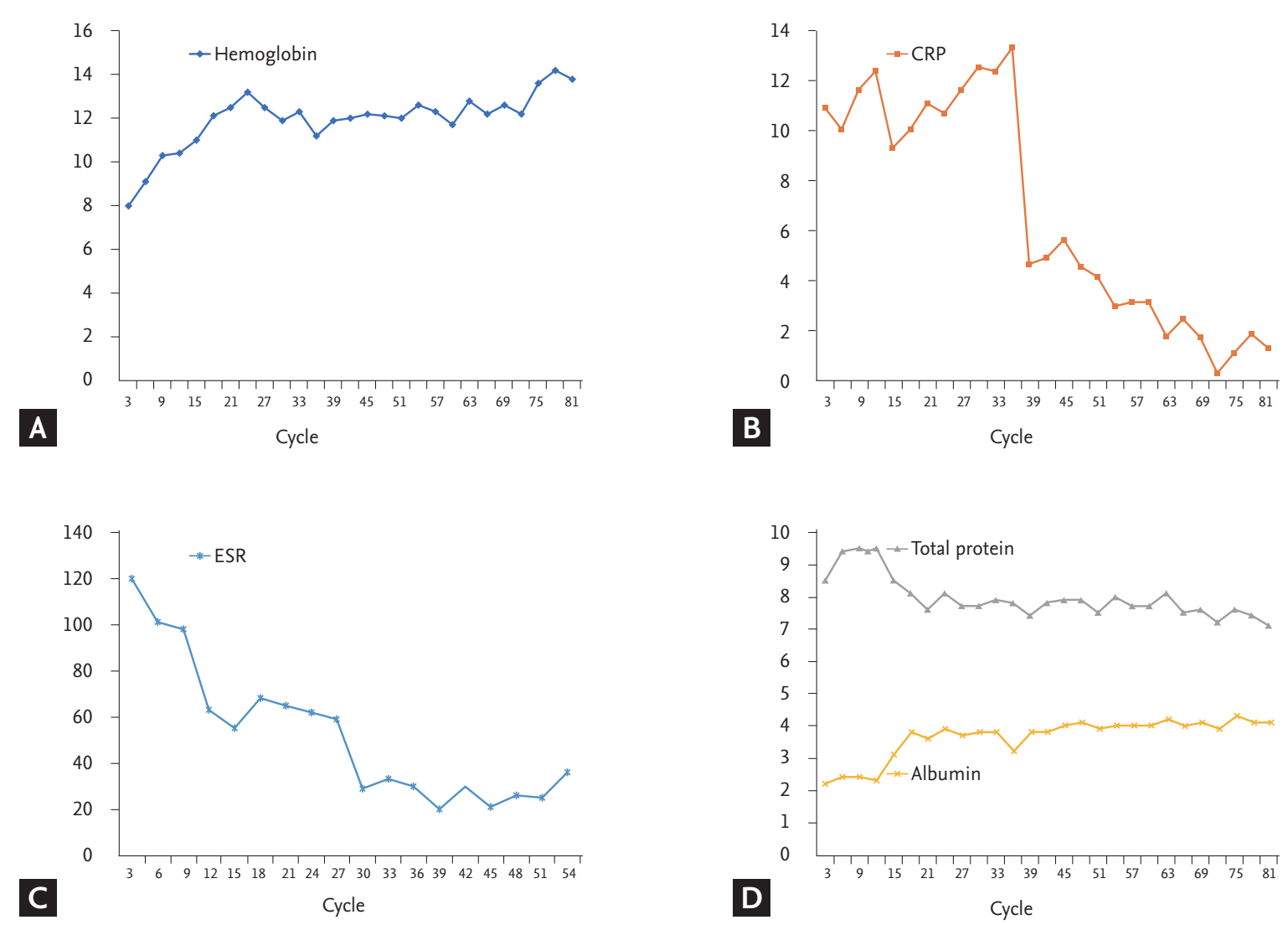

Figure 2. (A) Hemoglobin, (B) serum C-reactive protein (CRP), (C) erythrocyte sedimentation rate (ESR) values, and (D) total serum protein, and albumin levels in a patient who underwent long-term treatment with siltuximab.

spective design and a small sample size. First, the treatment responses and symptomatic changes in particular were not assessed as rigorously as in a prospective study. Consequently, our findings with respect to treatment response and iMCD progression are less reliable and may not be generalizable to other populations. Second, owing to the retrospective design, a possibility of bias remains. Third, in the period when these patients underwent treatment, the treatment of $\mathrm{MCD}$ was a challenge, and there were no evidence-based consensus guidelines or response evaluation criteria. Finally, although they represent a potential response criterion, the levels of IL-6, the key pathognomonic cytokine in $\mathrm{MCD}$, were not routinely assessed.

Although the Castleman Disease Collaborative Network has recently updated its guidelines [14], there is still no consensus on the cessation of administration of immunomodulatory agents with or without steroids. In our experience, laboratory values in these patients re- cover significantly after four cycles ( 3 months) of siltuximab, reaching stable normal levels after about 80 cycles (6o months) of maintenance treatment (Fig. 2). We speculate that radiologic CR should be a deciding factor for withholding siltuximab maintenance treatment because many real-world cases have shown delayed radiologic responses long after symptomatic relief and laboratory value normalization. The decision to discontinue maintenance treatment is a particular challenge for clinicians, and prospective cohort studies with long-term follow-up are essential for addressing this issue. Furthermore, as the siltuximab maintenance can be repeated until progression or toxicity, dose modification schedule might be inevitable. Unfortunately, because most of the siltuximab-treated patients only suffered from minor manageable adverse events, we did not attempt to reduce dosage or modify the intervals for siltuximab injection and followed U.S. Food and Drug Administration approved standard dose. Further dose modification studies involv- 
ing a larger iMCD patient group will prove to be valuable. Because VEGF plays a major role in the development of constitutional symptoms $[12,14,16]$, the combination of a VEGF receptor inhibitor and siltuximab represents another viable therapeutic option in patients with moderate to severe symptomatic iMCD.

In conclusion, siltuximab with best supportive care improved the symptoms, laboratory values, and imaging findings in our hospital cohort of patients with symptomatic iMCD. Siltuximab demonstrated a favorable safety profile, and prolonged treatment with siltuximab was well-tolerated with no new or cumulative toxic effects or treatment discontinuations and few serious adverse events. Despite the small number of patients in this study, the results are encouraging and reinforce the potential of siltuximab in the first-line treatment of iMCD. Further studies are needed to elucidate the clinical outcomes and adverse events associated with siltuximab.

\section{KEY MESSAGE}

1. Siltuximab is a safe first-line treatment option for idiopathic multicentric Castleman disease (iMCD).

2. In this small cohort, long-term treatment with siltuximab was well-tolerated, with no unusual or cumulative toxicities; the number of serious adverse events was also low.

3. Triple benefits, in the form of improved clinical, laboratory, and radiologic parameters, were observed on treatment of $\mathrm{MMCD}$ with siltuximab.

\section{Conflict of interest}

No potential conflict of interest relevant to this article was reported.

\section{Acknowledgments}

This study was funded by a grant from the Korea Healthcare Technology R\&D Project, Ministry for Health, Welfare, and Family Affairs, Republic of Korea (HI14C3417), and the Basic Science Research Program of the National Research Foundation of Korea, which is funded by the Ministry of Education, Science, and Technology (HI14C3417).

\section{REFERENCES}

1. Castleman B, Iverson L, Menendez VP. Localized mediastinal lymphnode hyperplasia resembling thymoma. Cancer 1956;9:822-830.

2. Castleman B, Towne VW. Case records of the Massachusetts General Hospital; weekly clinicopathological exercises; founded by Richard C. Cabot. N Engl J Med 1954;251:396-400.

3. Talat N, Belgaumkar AP, Schulte KM. Surgery in Castleman's disease: a systematic review of 404 published cases. Ann Surg 2012;255:677-684.

4. Dispenzieri A, Armitage JO, Loe MJ, et al. The clinical spectrum of Castleman's disease. Am J Hematol 2012;87:997-1002.

5. Gaba AR, Stein RS, Sweet DL, Variakojis D. Multicentric giant lymph node hyperplasia. Am J Clin Pathol 1978;69:86-90.

6. Keller AR, Hochholzer L, Castleman B. Hyaline-vascular and plasma-cell types of giant lymph node hyperplasia of the mediastinum and other locations. Cancer 1972;29:670683.

7. Simpson D. Epidemiology of Castleman disease. Hematol Oncol Clin North Am 2018;32:1-10.

8. Casper C. The aetiology and management of Castleman disease at 50 years: translating pathophysiology to patient care. Br J Haematol 2005;129:3-17.

9. van Rhee F, Stone K, Szmania S, Barlogie B, Singh Z. Castleman disease in the 21st century: an update on diagnosis, assessment, and therapy. Clin Adv Hematol Oncol 2010;8:486-498.

10. Nishimoto N, Kanakura Y, Aozasa K, et al. Humanized anti-interleukin-6 receptor antibody treatment of multicentric Castleman disease. Blood 2005;106:2627-2632.

11. Fajgenbaum DC, van Rhee F, Nabel CS. HHV-8-negative, idiopathic multicentric Castleman disease: novel insights into biology, pathogenesis, and therapy. Blood 2014;123:2924-2933.

12. van Rhee F, Wong RS, Munshi N, et al. Siltuximab for multicentric Castleman's disease: a randomised, double-blind, placebo-controlled trial. Lancet Oncol 2014;15:966-974.

13. van Rhee F, Fayad L, Voorhees P, et al. Siltuximab, a novel anti-interleukin-6 monoclonal antibody, for Castleman's disease. J Clin Oncol 2010;28:3701-3708.

14. van Rhee F, Voorhees P, Dispenzieri A, et al. Interna- 
tional, evidence-based consensus treatment guidelines for idiopathic multicentric Castleman disease. Blood 2018;132:2115-2124.

15. Cheson BD, Pfistner B, Juweid ME, et al. Revised response criteria for malignant lymphoma. J Clin Oncol
2007;25:579-586.

16. Fajgenbaum DC. Novel insights and therapeutic approaches in idiopathic multicentric Castleman disease. Blood 2018;132:2323-2330. 